\title{
Face Recognition System on Mobile using Similarity Data Mining Technique
}

\author{
Chatklaw Jareanpon \\ POLAR LAB, Department of Computer Science, Faculty of Informatics, Mahasarakham University, 44150, \\ THAILAND
}

*Corresponding Author: chatklaw.j@gmail.com, chatklaw.j@msu.ac.th

\begin{abstract}
This paper proposed the mobile face recognition using data mining technique system. This system is able to help the police to find the suspects from the crime database using Smartphones. The police has to mark the 10 important points in face that are 2 of beginning of the eye lids, 2 of end of the eye lids, nose tip, 2 of center of pupils, 2 of end of mouths, and chin prominence. This system is divided into 2 main functions which are creating the database, and recognizing the face in database. From the recognition process, this research used the data mining technique called "K-nearest neighbor technique". Our experiment is tested on the 100 pictures students from Department of Computer Science, Faculty of Infomatics, Mahasarakham University. The experiments are divided into 2 tests which are normal user test and software developer test. The normal user test is tested by 5 users with 30 problems, and the average percentage of true detection is $60.664 \%$. The software developer is tested on 3 times of 100 problems, and average percentage of true detection is $65.667 \%$.
\end{abstract}

Keywords: Face recognition, Face masking, Data mining

\section{Introduction}

The face recognition is vital to many tasks such as Facebook face tag, face defendant for police, security for example access control buildings, ATM machines, Image database investigation, Smart card. The face recognition consists of 2 processes that are face detection and face recognition. Many research interested in this topics. Xiaoguang $^{(1)}$ proposed the image analysis for face recognition using model-based approaches including Elastic Bunch Graph matching. Rabia and Hamid ${ }^{(2)}$ proposed the survey of face recognition techniques. The two primary tasks of face recognition is verification (one-to-one matching), and identification (one-to-many-matching). The usually features used for face recognition is ratios of distance, areas and angles. Shang-Hung ${ }^{(3)}$ proposed an introduction to face recognition technology. The framework for face recognition system consisted of 4 main processes that are face detector, eye localizer, facial feature extractor, and face recognizer. Zhimin et $\mathrm{al}^{(4)}$ proposed the face recognition with learning-based descriptor. The components are forehead, left eyebrow, right eyebrow, left eye, right eye, nose, left cheek, right cheek, and mouth. Tim et $\mathrm{al}^{(5)}$ proposed the principles and methods for face recognition and face modelling using adding the active shape model, skin textures. Yoshihisa et $\mathrm{al}^{(6)}$ proposed the security management for mobile devise by face recognition using Bayes law. The feature is variation of genders and ages.

Apart from the previous research, this research tries to use the simple face recognition for mobile because of low processor. The user will touch the 10 importance points on face image. After that, the distance between that points will automatically calculate and send to the server. The server will recognize with the database using Similarity Analysis.

\section{Face Recognition System on Mobile using Similarity Data Mining Technique}

This system starts with capturing and making the image using smart device, and smart device will calculate the distance, send to server. The server will identify by 
comparing the similarity with the database. This system consisted of 4 steps that are Pre-processing, Feature Extraction, Model generating, and Evaluation as shown in Figure 1 and Figure 2.

\subsection{Pre-Processing}

This research interested to use in mobile. The normal resolution of image from mobile is 4 to 5 megapixel. Then, this research firstly collects the 100 peoples of 5 megapixel images in JPEG format. The image is in clearly white background.

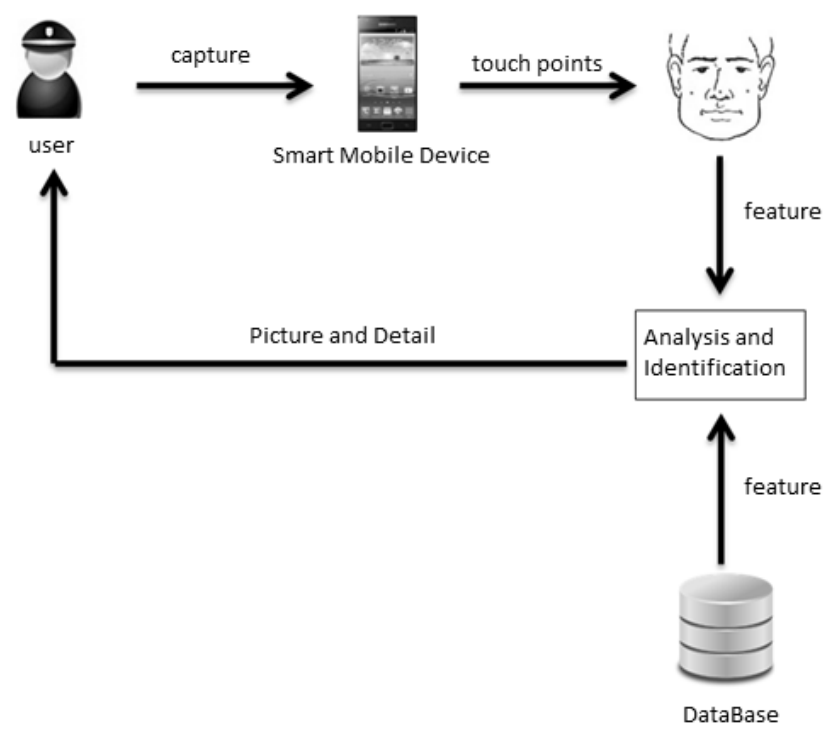

Fig. 1. Face Recognition System on Mobile using Data Mining Technique framework.

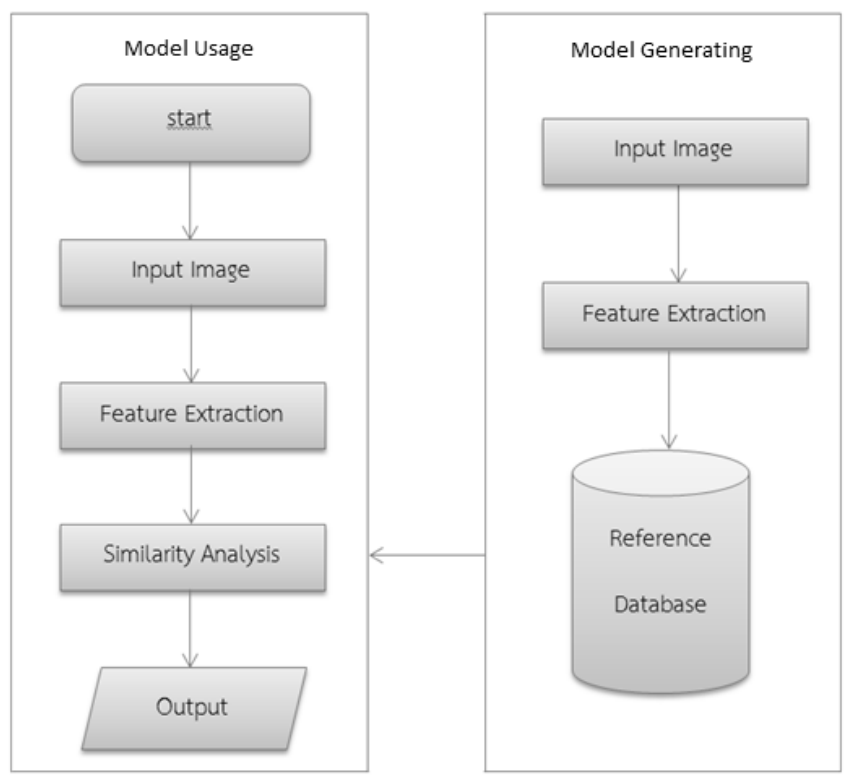

Fig. 2. Model Usage and Model Generating
2.1.1 Width and Height face ratio will detect by face tacking. The object in the various focal lengths such as short distance, long distance has different size. However, it is very difficult to calculate the focus length in mobile device, then this research selects to use the width and height of face ratio to normalize the position of $(\mathrm{x}, \mathrm{y})$. The $\mathrm{x}$ ' and $\mathrm{y}^{\prime}$ can be calculated by equation 1 and 2 . The width and height of face will show in Figure 3.

$$
x^{\prime}=\frac{x}{\text { width }} * 100
$$

$$
y^{\prime}=\frac{y}{\text { Height }} * 100
$$

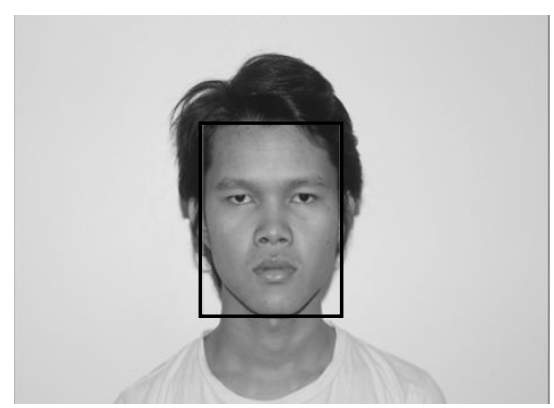

Fig. 3. Width and Height face ratio.

\subsection{Feature Extraction}

From Figure 3, the user must mark the 10 important points in face that are 2 of beginning of the eye lids, 2 of end of the eye lids, nose tip, 2 of center of pupils, 2 of end of mouths, and chin prominence as shown in Figure 4.
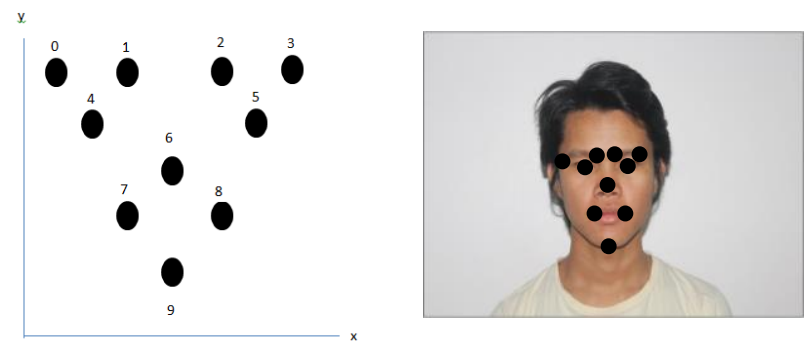

Fig. 4. The 10 important points in face.

The 10 positions will calculate the distance between all points by Euclidean (equation 3 ) that the features in this research are 45 features.

$$
\begin{aligned}
& \text { Euclidean Distance } \\
& =\sqrt{\left(x_{2}-x_{1}\right)^{2}+\left(y_{2}-y_{1}\right)^{2}}
\end{aligned}
$$


The example of the feature will show in Table 1.

Table 1. The features for each image.

\begin{tabular}{|c|c|c|c|c|c|c|c|c|c|}
\hline Image & $F 1$ & $F 2$ & $F 3$ & $F 4$ & $F 5$ & $F 6$ & $F 7$ & $F 8$ & $F 9$ \\
\hline \multirow{4}{*}{} & 5.61 & 10.82 & 16.74 & 4.07 & 13.22 & 10.72 & 13.89 & 16.94 & 20.54 \\
\cline { 2 - 11 } & $F 10$ & $F 11$ & $F 12$ & $F 13$ & $F 14$ & $F 15$ & $F 16$ & $F 17$ & $F 18$ \\
\cline { 2 - 11 } & 5.28 & 11.13 & 2.76 & 7.68 & 7.13 & 12.53 & 13.91 & 19.03 & 5.93 \\
\cline { 2 - 10 } & $F 19$ & $F 20$ & $F 21$ & $F 22$ & $F 23$ & $F 24$ & $F 25$ & $F 26$ & $F 27$ \\
\cline { 2 - 10 } & 7.52 & 2.92 & 7.44 & 13.6 & 12.55 & 19.02 & 13.22 & 3.83 & 10.3 \\
\cline { 2 - 10 } & $F 28$ & $F 29$ & $F 30$ & $F 31$ & $F 32$ & $F 33$ & $F 34$ & $F 35$ & $F 36$ \\
\cline { 2 - 10 } & 16.26 & 5.45 & 20.35 & 9.52 & 6.73 & 10.9 & 13.14 & 17.57 & 6.73 \\
\cline { 2 - 10 } & $F 37$ & $F 38$ & $F 39$ & $F 40$ & $F 41$ & $F 42$ & $F 43$ & $F 44$ & $F 45$ \\
\cline { 2 - 9 } & 13.34 & 10.83 & 17.89 & 6.51 & 6.58 & 12.33 & 5.62 & 6.72 & 7.3 \\
\hline
\end{tabular}

\subsection{Model Generating and Model Usage}

The simple recognition is try to compare the difference of the Euclidean distance between unknown and all record in database. This algorithm is similarity analysis called k-nearest neighbors algorithm ${ }^{(7-8)}$. This algorithm is simple, easy-to-implement, fast, and sophisticated machine learning methods. The algorithm can be summarized as:

1. A positive $\mathrm{k}$ is specified where $\mathrm{k}$ is the number of nearest output. The performance is primarily determined by the choice of $\mathrm{k}$. This research sets to 3 .

2. The identification of face recognition is calculated by equation 4 , then compare with the all records in database.

$Q_{n}-P_{n}$

$=\sqrt{\left(Q_{1}-P_{1}\right)^{2}+\left(Q_{2}-P_{2}\right)^{2}+\cdots+\left(Q_{45}-P_{45}\right)^{2}}$

where $\quad Q_{n}$ is the feature of unknown

$P_{n}$ is the feature in the database

$\mathrm{n}$ is the number of features.

3. Output is the k lowest distance record.

\subsection{Evaluation}

This paper will test the data using percentage of true detect normal calculated by equation 5 .

Percentage of true detect normal

$$
=\frac{T P}{\text { Total normal }} * 100
$$

where

TP is the true output

Total normal is the number of test.

\section{Experimental Result}

This research developed the algorithm on Android platform, and tested with the 2 experiments that are tested by user, and tested by developer. The main application will show in Figure 5(a-d). The Figure 5(a) is the main mobile application, and Figure 5(b) is the image with marking. The Figure 5(c) is the output and Figure 5(d) is the detail of selected output.

For tested by user, the 30 images will mark and test by 5 users, the average percentage of true detected rate is 60.66. The output will show in Figure 6. For tested by developer, the 100 images will test for 3 times, the average percentage of true detected rate is 65.66. The output will show in Figure 7.

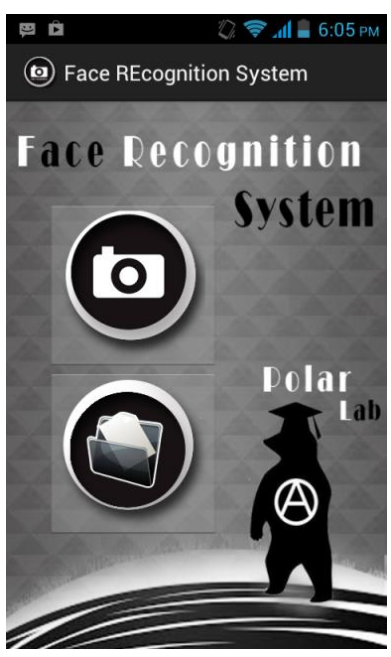

(a)
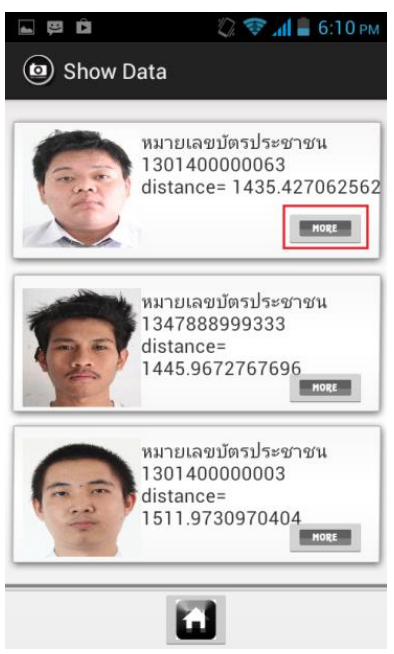

(c)

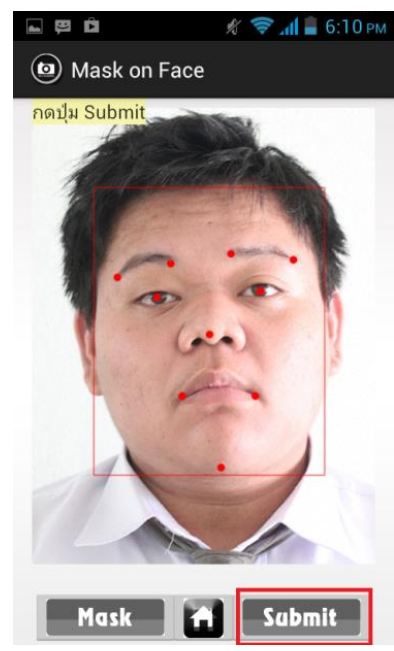

(b)

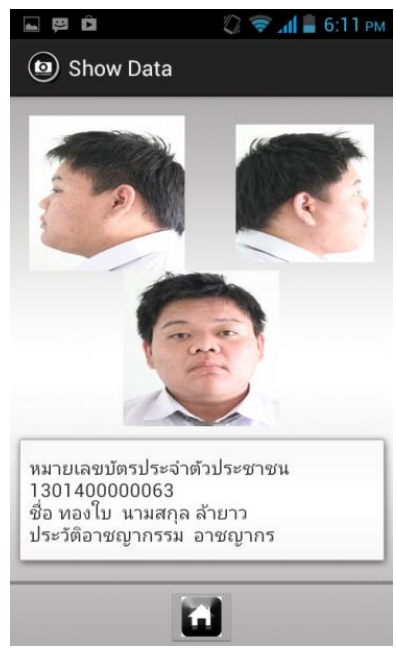

(d)
Fig. 5. Mobile application for testing the algorithm. 
The computational time of this algorithm is $O(n)$ when $\mathrm{n}$ is the number of training set.

\section{Discussion and Conclusions}

From the experimental result is able to summarize as follows: This similarity analysis using Euclidean distance algorithm is simple and able to use in mobile smart device.

The average percentage of true detection is equal to 60.66 tested by user, and equal to 65.66 tested by developer.

The difference of percentage of true detection comes from the experience of user. In the future work, the automatic making will use instead of manual marking. However, the algorithm of that must simple, and fast.

\section{Acknowledgment}

This research supported by Faculty of Informatics Mahasarakham University and Mahasarakham University.

\section{References}

(1) Xiaoguang Lu, Image Analysis for Face Recognition, personal notes, May 2003.

(2) Rabia Jafri and Hamid R. Arabnia, A Survey of Face Recognition Techniques, Journal of Information Processing Systems, Volumn 5, No.5, June 2009.

(3) Shang-Hung Lin, An Introduction to Face recognition Technology, Informaing Science Special Inssue on Multimedia Informaing Technologies-Part 2, Volume 3, No. 1, 2000.

(4) Zhimin $\mathrm{Cao}$ et al, Face recognition with Learning-based Descriptor, Proceeding of CVPR 2010 conference, 2010.

(5) Tim Rawlin son, Abhir Bhalerao, and Li Wang, Principles and Methods for Face Recognition and Face Modelling, In Handbook of Research on Computational Forensics, Digital Crime and Investigation: Methods and Solutions. Ed. C-T. Li. Chapter 3. Pages 53-78. 2010. ISBN 1605668362.

(6) Yoshihisa Ijuri, Miharu Sakuragi and Shihong Lao, Security Management for Mobile Devices by Face Recognition, Processing of the $7^{\text {th }}$ International Conference on Mobile Data Management (MDM'06), 2006.

(7) Sanjoy Dasgupta, Consistency of Nearest Neighbor Classification under Selective Sampling, The processing of JMLR Workshop and Conference, Volumn 23, 2012.

(8) Yang et al, IKNN: Informative K-Nearest Neighbor Pattern Classification, Lecture Notes in Computer Science: Knowledge Discovery in Databases: PKDD, Volumn 4702, pp. 248-264, 2007. 


\begin{tabular}{|l|l|l|l|c|}
\hline \multirow{2}{*}{ Input image } & \multicolumn{3}{|c|}{ Output } \\
\hline & The first nearest & The second nearest & The third nearest & \\
\hline Distance & & \\
\hline
\end{tabular}

Fig. 6. The example output tested by user. 


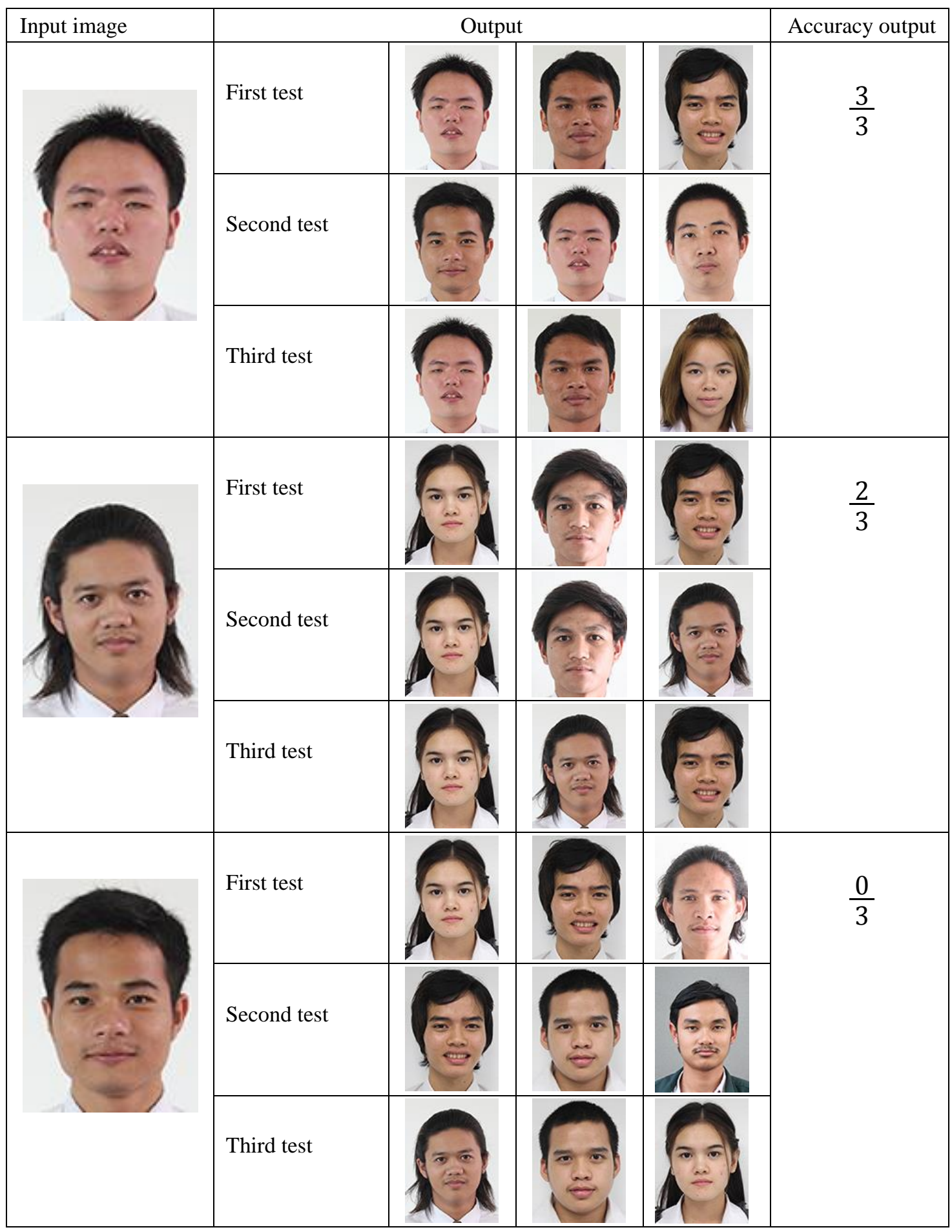

Fig. 7. The example output tested by developers. 